\title{
Impact of Process Variation on Self-Reference Sensing Scheme and Adaptive Current Modulation for Robust STTRAM Sensing
}

\author{
SEYEDHAMIDREZA MOTAMAN and SWAROOP GHOSH, Pennsylvania State University \\ JAYDEEP KULKARNI, Intel Labs
}

\begin{abstract}
Spin-Transfer-Torque RAM (STTRAM) is a promising technology for high-density on-chip cache due to low standby power and high speed. However, the process variation of the Magnetic Tunnel Junction (MTJ) and access transistor poses a serious challenge to sensing. Nondestructive sensing suffers from reference resistance variation, whereas destructive sensing suffers from failures due to unoptimized selection of data and reference currents. Furthermore, the sense speed is tightly coupled with the reference/data current requirement. In this work, we study the process variation effect on a self-reference sensing scheme to eliminate bit-to-bit process variation in MTJ resistance. Read current modulation is proposed to overcome the failures due to process variation. Simulation results reveal $<0.01 \%$ failures at the cost of $9 \mathrm{~ns}$ sense time and $190 \mathrm{uW}$ power consumption.
\end{abstract}

CCS Concepts: • Hardware $\rightarrow$ Integrated circuits; Robustness; Emerging technologies; Spintronics and magnetic technologies; Non-volatile memory; Failure recovery, maintenance and self-repair; Hardware reliability; Process, voltage and temperature variations;

Additional Key Words and Phrases: STTRAM, sense margin, self-reference, destructive sensing, process variation

\section{ACM Reference format:}

Seyedhamidreza Motaman, Swaroop Ghosh, and Jaydeep Kulkarni. 2017. Impact of Process-variation on SelfReference Sensing Scheme and Adaptive Current Modulation for Robust STTRAM Sensing. f. Emerg. Technol. Comput. Syst. 14, 1, Article 8 (October 2017), 17 pages.

https://doi.org/10.1145/3132577

\section{INTRODUCTION}

Spin-Torque Transfer Random Access Memory (STTRAM) (Hosomi et al. 2005) is a promising memory technology for embedded cache due to high density, low standby power, high speed, and CMOS-compatible voltage operation. STTRAM provides high density due to the 1T-1R structure and eliminates bitcell leakage due to the nonvolatile nature of the storage element, which is a magnetic tunnel junction (MTJ). The MTJ contains a free layer and a pinned magnetic layer (a cartoon is shown in Figure 1(a)). The resistance of the MTJ stack is high (low) if free layer magnetic orientation is antiparallel (parallel) compared to the fixed layer. The configuration of the MTJ can

This article is based on work supported by Semiconductor Research Corporation (\#2727.001) and National Science Foundation (CNS-1441757).

Authors' addresses: S. Motaman and S. Ghosh, Computer Science and Engineering Department, Pennsylvania State University, University Park-Pennsylvania; emails: \{sxm884, szg212\}@psu.edu; J. Kulkarni, Intel Labs, Hillsboro, OR; email: jaydeep.p.kulkarni@intel.com.

Permission to make digital or hard copies of all or part of this work for personal or classroom use is granted without fee provided that copies are not made or distributed for profit or commercial advantage and that copies bear this notice and the full citation on the first page. Copyrights for components of this work owned by others than ACM must be honored. Abstracting with credit is permitted. To copy otherwise, or republish, to post on servers or to redistribute to lists, requires prior specific permission and/or a fee. Request permissions from Permissions@acm.org.

(c) 2017 ACM 1550-4832/2017/10-ART8 $\$ 15.00$

https://doi.org/10.1145/3132577 


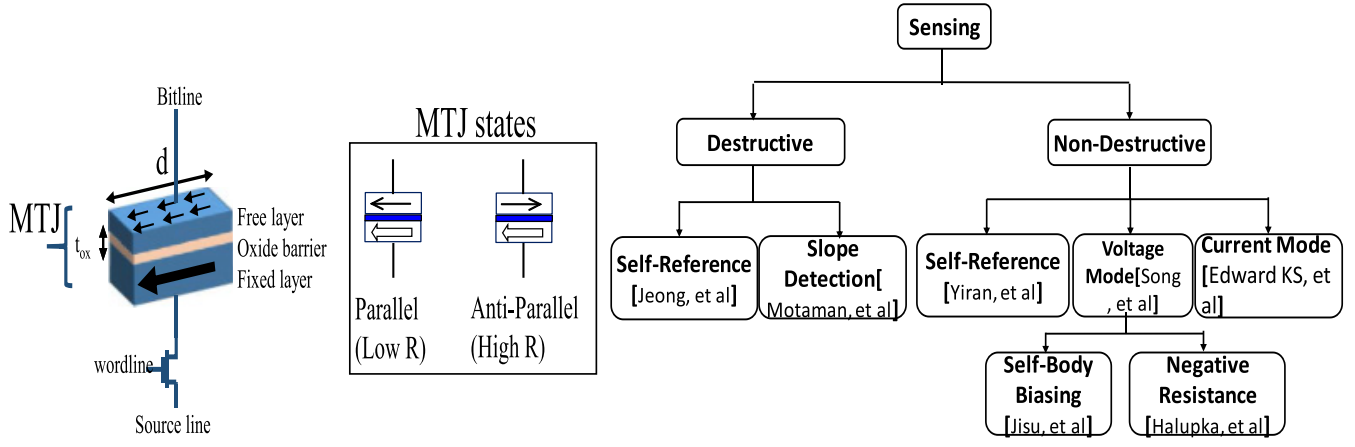

(a)

(b)

Fig. 1. (a) Schematic of STTRAM. (b) Taxonomy of STTRAM sensing schemes.

be changed from parallel to antiparallel (or vice versa) by injecting current from the source line to the bitline (or vice versa). The spin-torque transfer phenomena for reversal of magnetization in the free layer reduce the write power compared to conventional Magnetic Random Access Memory (MRAM), where the writing is field based. With the physical scaling of dimensions, the field needed to switch the free layer increases in MRAM. Current-induced switching makes STTRAM energy efficient compared to its MRAM counterpart. The memory array contains circuits to sense the orientation of the free magnetic layer compared to the fixed layer. The read operation is performed by sensing the orientation of the free layer with respect to the fixed layer in the form of resistance change.

The crucial challenges involved in STTRAM are high write current, long write time, and poor sense margin (SM). MTJ resistance depends on various factors including barrier thickness, free and fix layer area, shape, and thickness. Several methods have been proposed in the past to reduce the write current by minimizing the number of writes (Zhou et al. 2009; Rasquinha et al. 2010). Device architecture space is explored to reduce write power by lowering the thermal energy barrier by trading nonvolatility (Xu et al. 2010 and Smullen et al 2011). Hybrid cache architecture has been proposed to reduce write power (Imani et al 2016). A Dead Write Prediction Assisted STTRAM cache architecture, which predicts and bypasses dead writes for saving write energy, has been proposed in Junwhan et al. (2014). A hybrid cache architecture composed of magnetic RAM (MRAM) and SRAM, which exploits narrow-bit data to save energy, is proposed in Imani et al. (2016). Interesting circuit architecture methods (e.g., balanced write, flipped MTJ with sequential tag-data access and partial line update, $2 \mathrm{~T}-1 \mathrm{R}$ with negative bitline, read optimized bitcell with stretched write cycle ( $\mathrm{Li}$ et al. 2010; Kim et al. 2012; Lee et al. 2012)) have also been proposed to eliminate write power and write time challenges associated with STTRAM. In Motaman et al. (2014), simultaneous sizing, reference voltage, and clamp voltage biasing have been exploited to improve the robustness and testability of STTRAM arrays.

Sense margin of STTRAM depends on TMR (Tunnel Magnetic Resistance), which is defined as $100 \%$ * $\left(R_{H}-R_{L} / R_{L}\right)$, where $R_{L}$ and $R_{H}$ are low and high resistance, respectively. Due to poor TMR, the voltage/current differential between $\mathrm{R}_{\mathrm{H}}$ and $\mathrm{R}_{\mathrm{L}}$ decreases, which degrades the SM. Besides, SM0 (sense "0" margin) and SM1 (sense "1" margin) are not identical (Emre et al. 2012).

Figure 1(b) shows the taxonomy of various sensing techniques. STTRAM sensing can be broadly categorized into destructive and nondestructive sensing. Several techniques have been proposed under nondestructive sensing. A nondestructive voltage sensing and a sizing methodology to 
improve the SM of MRAM arrays have been proposed in Song et al. (2011). Source degeneration and a balanced reference scheme are proposed in Seong-Ook et al. (2011) and Jisu et al. (2012) to reduce large sense margin variation. A self-body biasing technique has been proposed in Jisu et al. (2014) to resolve the small sense margin issue in a conventional voltage sensing scheme. A negative resistance read and write technique has been described in Halupka et al. (2010) to eliminate read disturb and reduce the write power. Reference voltage $\left(\mathrm{V}_{\text {ref }}\right)$ biasing has been explored in Ren et al. (2012) to shift margins between polarities to improve the robustness. A nondestructive current mode sensing scheme using a current conveyor has been proposed in Edward et al. (2004). One of the primary challenges with reference-based design is the susceptibility of reference voltage to process variation.

In Yiran et al. (2012), a nondestructive self-reference sensing scheme has been proposed by leveraging the dependencies of the high- and low-resistance state of MTJ on the cell current amplitude. Even though this scheme reduces the read latency and power by eliminating two write steps, the sense margin is much smaller than that of the self-reference scheme (Jeong et al. 2003) and conventional nondestructive voltage sensing (Song et al. 2011).

Under destructive sensing, the self-reference sensing scheme has been proposed in Jeong et al. (2003) to eliminate bit-to-bit process variation in MTJ resistance. Sensing is performed by first storing the voltage of the MTJ by passing a current $\left(\mathrm{I}_{1}\right)$ and then after a time interval storing a reference voltage of the same MTJ after passing a current $\left(\mathrm{I}_{2}\right)$. The variation in MTJ resistance can be eliminated by a self-reference sensing scheme. Although this mechanism incurs high power consumption and long read latency due to two write steps, it provides a high sense margin and eliminates the need of reference voltage. The slope detection technique has been proposed in Motaman et al. (2015) to exploit MTJ resistance switching from a high to low state. In this technique, ramp current is injected into bitcell, which results in a ramp voltage. The slope of voltage changes from positive to negative if the MTJ resistance is switched from a high to low resistance state. Therefore, a sensing problem can be simplified to slope detection. If a negative slope is detected, then the data is sensed as " 1 ,"; otherwise, the data is sensed as " 0. "

In this article, we study the impact of process variation on a self-reference sensing scheme (Jeong et al. 2003). Our investigation reveals that unoptimized selection of reference and data current can result in significant sensing failures. We also show that sense speed is tightly coupled with reference and data current requirements and sense failures. We propose a reference and data current modulation technique to minimize the sense failures. The proposed sensing can be exploited in applications in which the data will be read one time only and can be discarded afterward such as Network on Chip (NOC) buffers and FIFO buffers. Moreover, it can be used in video streaming applications for buffering each video frame where data will be read only one time. In these applications, it is not required to write the data back after reading; therefore, the latency of the proposed technique is comparable to conventional nondestructive sensing, while the robustness under process variation is improved significantly.

The article is organized as follows: In Section 2, we describe nondestructive and destructive sensing schemes and the design challenges. In Section 3, the process variation effect on sense failures and selection of data and reference current is investigated. The impact of sense speed on sense failures is also presented in this section. Read current modulation to reduce sensing failures is discussed in Section 4. Conclusions are drawn in Section 5.

\section{RELATED WORK AND ANALYSIS}

In this section, we discuss the conventional nondestructive sensing scheme and the design challenges. Next, we explain the destructive self-reference sensing scheme and describe the challenges. We used 22nm predictive technology for designing the proposed sensing scheme. 
Table 1. Parameters Used for Process Variation Study

\begin{tabular}{|l|c|c|l|}
\hline Device & Parameter & Mean & Std. Dev. \\
\hline Transistor & $\mathrm{V}_{\mathrm{TH}}$ & 0.5 & $50 \mathrm{mV}$ \\
\hline MTJ & $\begin{array}{c}\text { MgO } \\
\text { Thickness }\end{array}$ & $1.1 \mathrm{~nm}$ & $3 \%$ \\
\hline Shape Area & $\mathrm{Pi}^{*} 625 \mathrm{~nm}^{2}$ & $5 \%$ \\
\hline
\end{tabular}

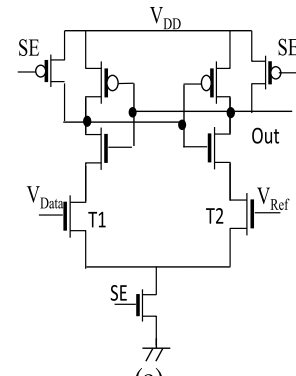

(a)

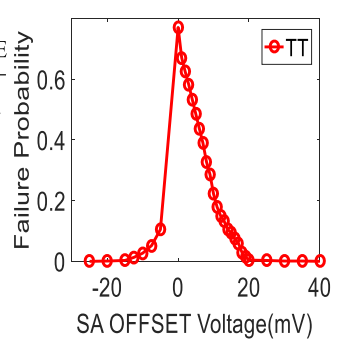

(b)

Fig. 2. (a) Sense amplifier circuit. (b) Senseamp offset voltage distribution for 1,000-point MonteCarlo simulations.

\subsection{Nondestructive Voltage Sensing Scheme (Song et al. 2011)}

The purpose of the sense circuit is to identify the resistance of the data MTJ. In order to make the comparison, data MTJ resistance is compared against reference MTJ resistance (which is an average of fixed high and low MTJ resistances). Figure 3(a) shows the typical voltage sensing where a reference current is injected in both data legs and reference legs and the resulting voltage is compared by a voltage sense amplifier (SA). Poor sense margin can result in wrong interpretation of the MTJ state. For example, if the offset voltage of the sense amplifier is $+/-25 \mathrm{mV}$, a sense margin of $25 \mathrm{mV}$ can be read as either "0" or " 1. "

The SA offset voltage ( $\mathrm{V}_{\text {SA os }}$ ) depends on sense time and SA size since increasing transistor size decreases the transistor threshold voltage variation (Equation (1)). We design the SA in such a way to reduce the offset while meeting the area and delay requirements. We considered an SA

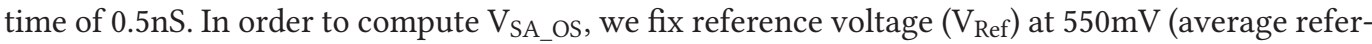
ence voltage generated by sense circuit) and sweep $V_{\text {Data }}$ (Figure 2(a)). For each sweep, 1000-point Monte-Carlo simulation is performed and SA failure distribution is shown in Figure 2(b). The sense amplifier output node (OUT) is initially precharged to "1." If $\mathrm{V}_{\mathrm{Ref}}+\mathrm{V}_{\mathrm{SA}}$ OFF $>\mathrm{V}_{\text {Data }}$, node OUT is pulled down to " 0 ." Since node OUT is initially precharged to " 1 ," the failure probability of sensing " 0 " (where senseamp offset $>0$ ) is greater than sensing " 1 " (where senseamp offset $<0$ ). As shown in Figure 2(b), sense amplifiers sense data correctly by providing $40 \mathrm{mV}$ SM for sensing "0" and $-25 \mathrm{mV}$ SM for sensing “1.”

\section{Impact of Process Variation}

The MTJ model (Fong et al. 2013) is used in order to perform process variation analysis. Process variations for read operations are modeled by incorporating variations in MTJ as well as access transistors. For MTJ, we have assumed tunnel oxide barrier and surface area variations. The variations in the access transistor are lumped in threshold voltage fluctuations. The mean and standard deviation of these parameters are provided in Table 1. The retention time of MTJ we used is about 1 year in the standby mode. In nondestructive sensing, resistance of data MTJ is compared against the resistance of reference MTJ to determine the bitcell content. Therefore, reference resistance as well as data resistance variation may result in wrong interpretation of bitcell content. Data0, Data1, and reference voltage distributions for 10,000 Monte-Carlo points is shown in Figure 3(b). Sensing error occurs where reference voltage overlaps with Data0/1 voltage. The process variation impact on the nondestructive voltage sensing scheme has been investigated in Jisu et al. (2012); however, 


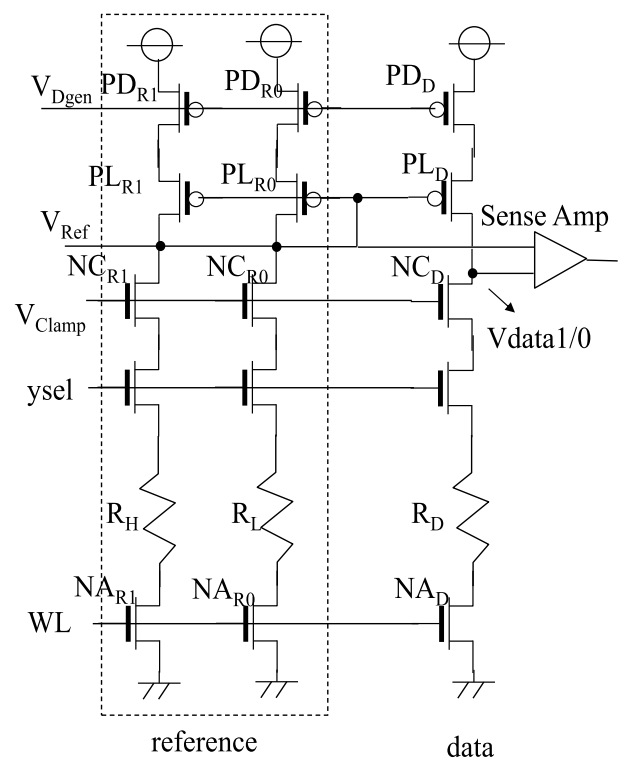

(a)

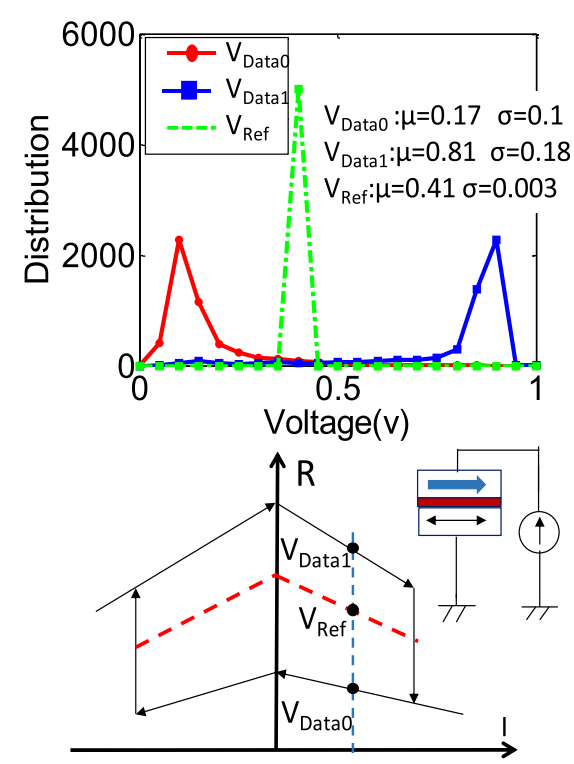

(b)

Fig. 3. (a) Nondestructive sensing scheme, (b) Data0 reference and Data1 voltage distributions.

one sigma of MTJ resistance distribution is assumed as 4\%, which is not realistic for large arrays. We consider MTJ area and oxide thickness variation (Dongsoo et al. 2014; Yaojun et al. 2011; Jing et al. 2008) using the MTJ model (Fong et al. 2013).

Two critical transistors in the STTRAM sense circuit are the PMOS load (PL) and NMOS clamp (NC) (Figure 3(a)). The clamp voltage and clamp transistor size set the current in the leg. The load transistor sets the output voltage (where the NMOS and PMOS drain currents intersect). The mismatch between matched-pair transistors in the sense circuit degrades the sense margin. Since the size of PL is smaller than the NC transistor, the sense margin is most sensitive to mismatch between the PL transistors of reference legs $\left(\mathrm{PL}_{\mathrm{R}}\right)$ and that of data legs $\left(\mathrm{PL}_{\mathrm{D}}\right)$. One sigma of $\mathrm{V}_{\mathrm{T}}$ variation is given by the following equation (Pelgrom et al. 1989):

$$
\sigma_{V_{T}}=\frac{A_{V_{T}}}{\sqrt{W . L}}
$$

where $\mathrm{W}$ and $\mathrm{L}$ are the width and length of the transistor, respectively, and $A_{V_{T}}$ is the pelgrom coefficient. The sense circuit is designed to reduce the impact of process variation on SM. This goal is achieved by increasing the width and length of PL transistors to reduce the $\mathrm{V}_{\mathrm{T}}$ mismatch between $\mathrm{PL}_{\mathrm{D}}$ and $\mathrm{PL}_{\mathrm{R}}$ and optimizing other design parameters such as $\mathrm{NC}$ width, $\mathrm{V}_{\text {clamp }}$, and $\mathrm{V}_{\text {Ref }}$ to maximize both SM0 and SM1. However, increasing the PL width/length increases area overhead and coupling capacitance between the reference and data leg. Since many data legs share one reference leg (32 in this analysis), the coupling noise from the gate and drain of $\mathrm{PL}_{\mathrm{D}}$ to the gate node of $\mathrm{PL}_{\mathrm{R}}$ degrades the sense speed. We pick $\mathrm{W}_{\mathrm{PLD}} / \mathrm{L}_{\mathrm{PLD}}$ to reduce $\mathrm{V}_{\mathrm{T}}$ mismatch as well as coupling noise.

Since NC and PL transistors in the data leg are shared in a global column (each global column consists of eight local columns) and reference legs are shared among 32 local columns, their sigma is assumed to be $\frac{1}{\sqrt{8}}$ and $\frac{1}{\sqrt{32}}$ of sigma, calculated by Equation (1). Distributions of SM0 and SM1 for 


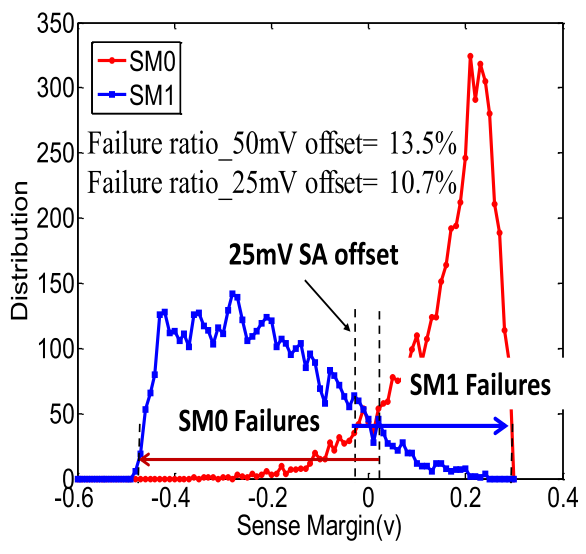

(a)

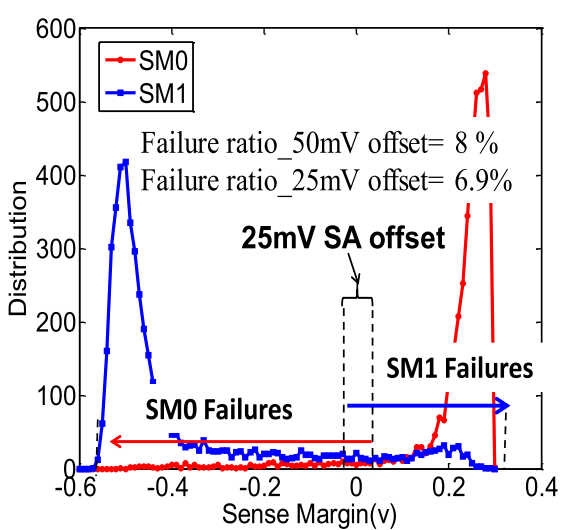

(b)

Fig. 4. SM0 and SM1 distribution for 10000 Monte-Carlo points: (a) original scheme (Song et al. 2011) and (b) with source degeneration (Jisu et al. 2012).

10,000 Monte-Carlo points are depicted in Figure 4(a). Simulations reveal that $10 \%$ of bitcells fail sensing due to SM0 failures and $11.2 \%$ fail due to SM1 failures, which results in $10.7 \%$ total failures for $25 \mathrm{mV}$ SA offset. It is evident that conventional nondestructive sensing is prone to process variation.

To reduce the large sense margin variation, the source degeneration scheme is used with a longer channel length for PL transistors (Seong-Ook et al. 2011). Source degeneration PMOS (PD) is inserted between $\mathrm{V}_{\mathrm{DD}}$ and source of PL transistors to reduce current variation and increase effective resistance, which result in an improved sense margin. We follow the same procedure as proposed in Jisu et al. (2012) to optimize the design. Figure 4(b) shows SM0 and SM1 distributions for 10,000 Monte-Carlo points with a source degeneration scheme. The simulation reveals $5.8 \%$ SM0 failures and $8 \%$ SM1 failures, which result in $6.9 \%$ total failures for $25 \mathrm{mV}$ SA offset. Although source degeneration reduces the sense failures, they are still significant, which underscores the need for a self-reference scheme to eliminate bit-to-bit variation as well as the mismatch between matched-pair transistors in data and reference legs.

\subsection{Destructive Self-Reference Sensing Scheme (Jeong et al. 2003)}

In self-reference sensing, voltage generated by the data current across the MTJ and the voltage generated by a reference current across the same MTJ are compared. Therefore, the bit-to-bit variation in MTJ resistance is eliminated. The self-reference sensing scheme works as follows (Figure 5):

(1) A read current $I_{\text {Data }}$ is injected into the bitcell and the resulting voltage is stored in a capacitor $\mathrm{C}_{1}$. The voltage could be $\mathrm{V}_{\text {DataH }}\left(\mathrm{V}_{\text {DataL }}\right)$ if the MTJ resistance is high (low).

(2) A zero is written into the MTJ.

(3) Another read current $I_{\text {Ref }}$ is applied to generate voltage $V_{\text {Ref }}$, which is stored in capacitor $\mathrm{C}_{2}$.

(4) $V_{\text {Data }}$ and $V_{\text {Ref }}$ are compared to determine the bitcell content.

(5) The read data is written back to the MTJ.

Consideration for process variations is important to determine appropriate values of $\mathrm{I}_{\mathrm{Ref}}$ and $\mathrm{I}_{\text {Data }}$. The I-R curves of the two MTJs under process variation are shown in Figure 6 . The $\mathrm{I}_{\text {Data }}$ and $\mathrm{I}_{\text {Ref }}$ must be selected in such a way to ensure SM0 is positive and SM1 is negative. Therefore, the 


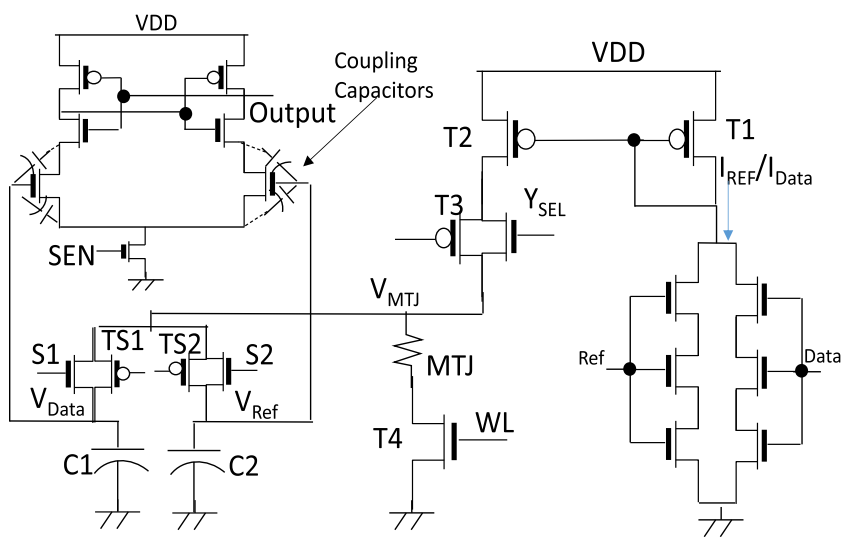

(a)

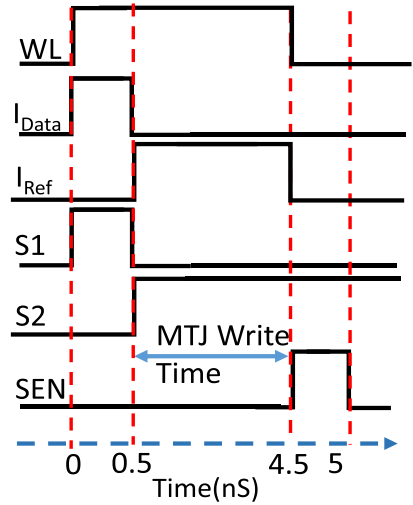

(b)

Fig. 5. (a) Self-reference sensing scheme. (b) Sense circuit timing diagram.

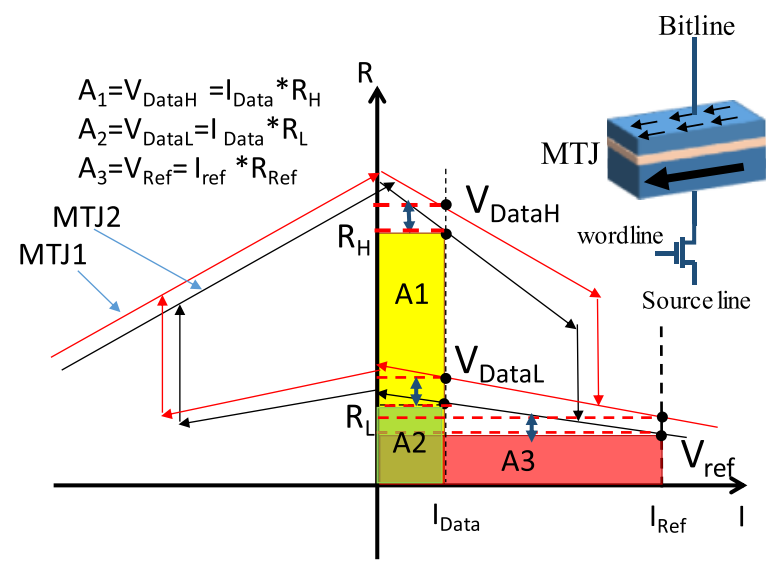

Fig. 6. I-R characteristics of the two MTJs under process variation. A variation in resistance can change the sense margin.

area A3 (which is essentially voltage) should be greater than A2 and less than A1, which results in the following inequalities:

$$
\begin{aligned}
& V_{\text {DataL }}<V_{\text {Ref }}<V_{\text {DataH }} \\
& S M 0=V_{\text {Ref }}-V_{\text {DataL }}>0 \\
& S M 1=V_{\text {Ref }}-V_{\text {DataH }}<0
\end{aligned}
$$

Challenges: Determining optimum $\mathrm{I}_{\mathrm{Ref}}$ and $\mathrm{I}_{\text {Data }}$ to achieve the best sense margin for each bitcell under process variation is not possible from an implementation standpoint. Choosing $\mathrm{I}_{\text {Ref }}$ and $\mathrm{I}_{\text {Data }}$ to maximize the sense margin for the majority of the bitcells is challenging as well. Figure 6 shows the voltage fluctuation in two MTJs due to process variation. The area under A1, A2, and A3, which is $\mathrm{V}_{\text {DataH }}, \mathrm{V}_{\mathrm{Ref}}$, and $\mathrm{V}_{\text {DataL }}$, respectively, fluctuates due to process variation. Therefore, determining corresponding data and reference currents to maximize sense margin for all bitcells is challenging.

Sense Time: Self-reference sensing is performed in five steps. In our design, we merge the second and third steps by writing the MTJ while applying the reference current. Next, we compare the 


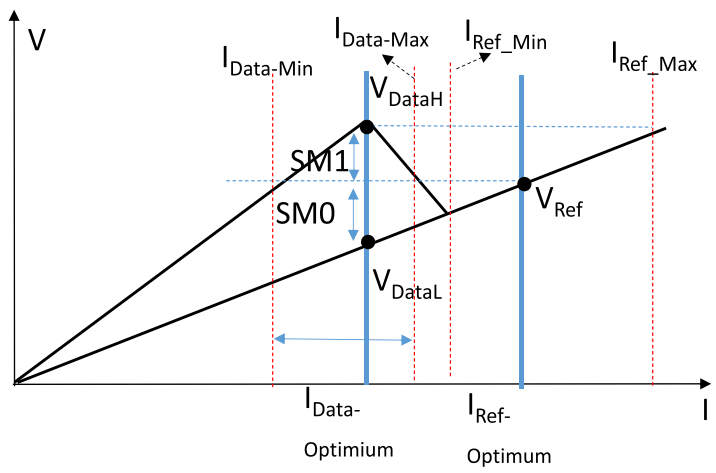

Fig. 7. V-I curves of an MTJ with high and low resistance states initially. The voltage increases monotonically when the initial resistance is low. For the high initial resistance, the voltage swings back after the MTJ state is flipped to low. Note that $I_{\text {Ref }}$ should be (i) greater than $I_{\text {Ref-Min }}$ to ensure writing a zero to the MTJ and (ii) less than $I_{\text {Ref-Max }}$ to obtain negative SM1. I Data should be (i) greater than I Data-Min to achieve negative SM1 and (ii) less than IData-Max to achieve positive SM0.

capacitor values using a sense amplifier. Therefore, sense time is the cumulative time required to perform these three steps plus the time needed to write the original data back to the MTJ. The timing diagram of sensing is depicted in Figure 5(b). First, a data current applied for a period of time and its value are stored in the first capacitor by turning ON switch TS1. The pulse width of signal S1 depends on the RC delay of TS1 and capacitor $\mathrm{C}_{1}$. We determine the S1 pulse width as the time when $\mathrm{V}_{\text {Data }}$ is $95 \%$ of $\mathrm{V}_{\mathrm{MTJ}}$. In our simulations, the worst-case sense time is $0.5 \mathrm{~ns}$ (time required for storing $\mathrm{V}_{\text {Data }}$ ) $+4 \mathrm{~ns}$ (time required to flip the MTJ and store $\mathrm{V}_{\text {Ref }}$ ) $+0.5 \mathrm{~ns}$ (time required for sense amplifier) $+4 \mathrm{~ns}$ (time required to write the original value back to the MTJ), which leads to 9 ns sense time.

\section{PROCESS VARIATION EFFECT ON SELF-REFERENCE SENSING}

In this section, the impact of process variation on optimized selection of data and reference current is investigated. In addition, the interaction between process-variation-induced sense margin fluctuation and sensing speed is also explored.

\subsection{Simulation Setup}

Process variation analysis is important to determine the $\mathrm{I}_{\text {Ref }}$ and $\mathrm{I}_{\text {Data }}$, which provides positive and negative sense margins for SM0 and SM1, respectively. The mean and standard deviation of these parameters are provided in Table 1 . The process variation analysis has already been discussed in Dongsoo et al. (2014) and Yaojun et al. (2011) in detail.

\subsection{METHODOLOGY}

To understand the impact of process variation on data and reference current requirements, we sweep the bitcell current from bitline to source line. If the bitcell state is low, the voltage across MTJ increases monotonically with current. However, if the bitcell state is high, it switches to a low-resistance state beyond the critical current. Therefore, the voltage changes from high to low. As depicted in Figure 7, $\mathrm{I}_{\text {Ref }}$ and $\mathrm{I}_{\text {Data }}$ should be chosen carefully to ensure + ve SM for high data and -ve $S M$ for low data. Therefore, $V_{\text {Ref }}$ should be greater than $\mathrm{V}_{\text {DataL }}$ and less than $\mathrm{V}_{\text {DataH }}$, where $\mathrm{V}_{\text {DataL }}\left(\mathrm{V}_{\text {DataH }}\right)$ is the voltage across the MTJ when the stored data is low (high). 


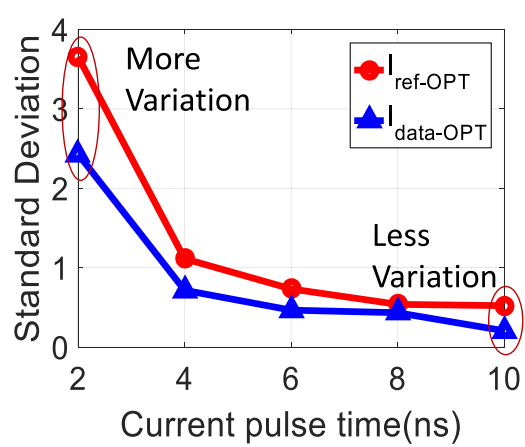

(a)

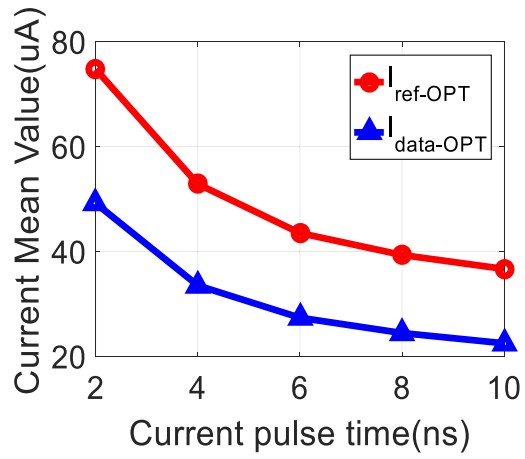

(b)

Fig. 8. Optimum reference and data current: (a) variation and (b) mean value with respect to reference current pulse time.

As shown in Figure 7, the optimum $\mathrm{I}_{\text {Data }}$ is the current that maximizes SM0 + SM1 (i.e., the current where $\mathrm{V}_{\text {DataH }}$ is maximum). Optimum $\mathrm{I}_{\text {Ref }}$ is chosen to equalize SM0 and SM1. $\mathrm{I}_{\text {Ref }}$ must be less than $\mathrm{I}_{\text {Ref-Max }}$, which is the current when SM1 vanishes. Additionally, $\mathrm{I}_{\text {Ref }}$ must be chosen to ensure all MTJs switch from a high to low resistance state. Therefore, $\mathrm{I}_{\mathrm{Ref}}$ must be greater than $\mathrm{I}_{\text {Ref-Min }}$ of all bits:

$$
\operatorname{Max}_{i \in\{b i t s\}}\left(I_{R e f-M i n}(i)\right)<I_{R e f}<\operatorname{Min}_{i \in\{b i t s\}}\left(I_{R e f-M a x}(i)\right) .
$$

It is also evident that $\mathrm{I}_{\text {Data }}$ must be less than $\mathrm{I}_{\text {Data-Max }}$ (i.e., the current after which SM1 is negative) and greater than $\mathrm{I}_{\text {Data-Min }}$ (i.e., the current below which SM1 is negative) of all bits in order to sense all bits correctly, which results in the following inequality:

$$
\operatorname{Max}_{i \in\{b i t s\}}\left(I_{\text {Data-Min }}(i)\right)<I_{\text {Data }}<\operatorname{Min}_{i \in\{b i t s\}}\left(I_{\text {Data-Max }}(i)\right) .
$$

\subsection{Reference and Data Current Variation and Sense Speed}

The analysis presented in Section 3.2 considers DC values of reference and data current. However, the sensing is also dependent on the amount of time for which the current is applied. Higher current flips MTJ faster, whereas lower current takes longer to flip. Furthermore, longer sense time eventually flips the bits when the write current is around the critical current of MTJ. A tighter sense time for the same current may not flip the bit at all. Therefore, faster sense time is associated with higher current and is sensitive to changes in MTJ write time due to process variations. The relaxed sense time experiences less variation in optimum reference and data current. Although robust to variation, longer sense time adversely affects the memory latency.

Figures $8(\mathrm{a})$ and $8(\mathrm{~b})$ show the mean and sigma of reference and data current for different amounts of sense period for 2,000 runs of Monte-Carlo. It can be observed that a faster-current pulse requires higher mean current to perform sensing. Furthermore, the faster sensing also results in more spread of data and reference current than wider pulse. Figures 9(a) to 9(c) capture the variation in data and reference current for pulse widths of $2 \mathrm{~ns}, 5 \mathrm{~ns}$, and $10 \mathrm{~ns}$. It is evident that a longer current pulse time results in less variation in data current (less variation in Data $\mathrm{H}$ peak voltage). 


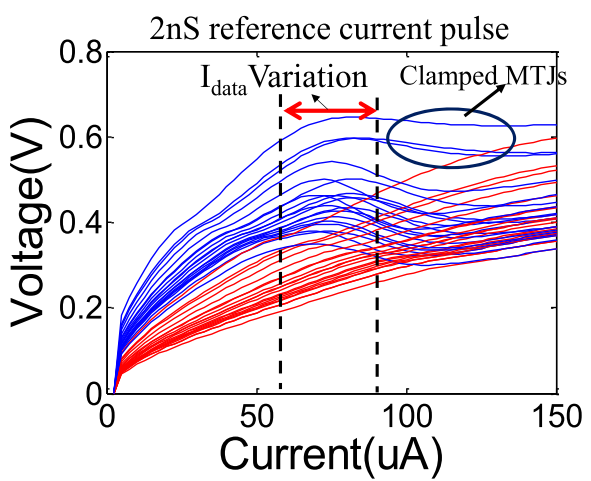

(a)

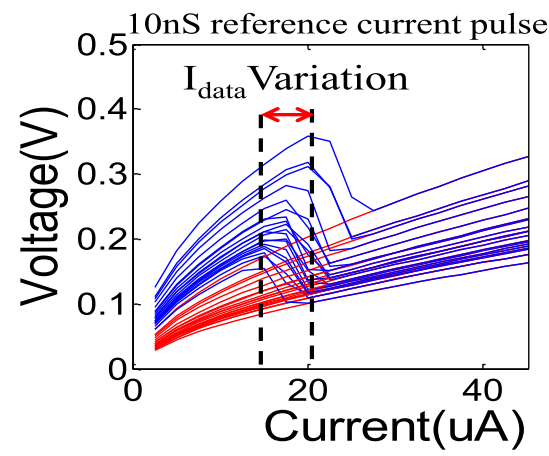

(c)

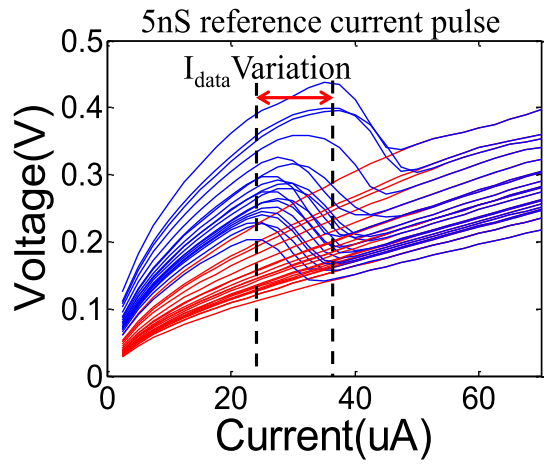

(b)

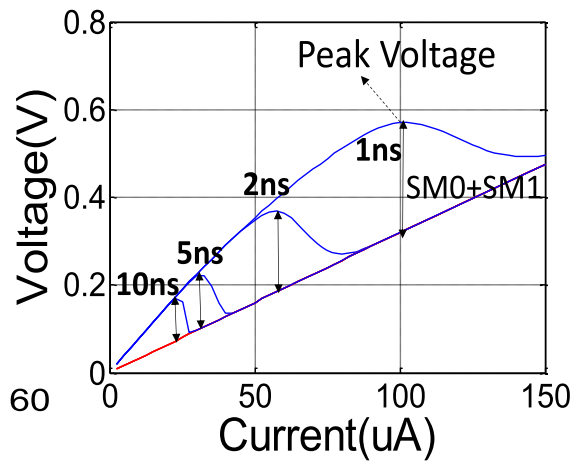

(d)

Fig. 9. Optimum data current variation with respect to reference current pulse: (a) 2ns, (b) 5ns, (c) 10ns, and (d) MTJ flipping current and voltage peak with respect to different current pulse time.

\subsection{Sense Margin and Sense Speed}

A faster sense period results in more variation in optimum reference and data current; therefore, the sense margin experiences more variation. Interestingly, the faster sensing requires higher reference and data current to flip the bit, which provides higher voltage and better sense margin (i.e., higher $|\mathrm{SM} 0|+|\mathrm{SM} 1|)$. This is pictorially shown in Figure 9(d). However, the voltage across bitcell $\left(\mathrm{V}_{\mathrm{MTJ}}\right)$ is clamped at $\mathrm{V}_{\mathrm{DD}}-\mathrm{V}_{\mathrm{od} 2}$ (overdrive voltage of transistor $\left.\mathrm{T} 2\right)-\mathrm{V}_{\mathrm{od} 3}$ (overdrive voltage of transistor T3) in Figure 5(a). Therefore, even with high current, the voltage across bitcell cannot increase beyond a certain voltage, limiting the sense margin. As shown in Figure 9(a), some bitcells are clamped near $0.65 \mathrm{~V}$ and fail to be sensed correctly. Therefore, faster sensing speed is limited by circuit and MTJ constraints.

The slower sensing is robust to variation; however, it requires low current, which reduces the sense margin (Figure 9(d)). Note that it is not possible to improve the sense margin by increasing the reference/data current for slower sensing. This is true due to sense margin limitations set by Equations (3) and (4). Therefore, sense-speed-, sense-margin-, and process-variation-induced failures are coupled with each other.

\subsection{Simulation Results}

We have evaluated four cases for determining robust reference and data current: 


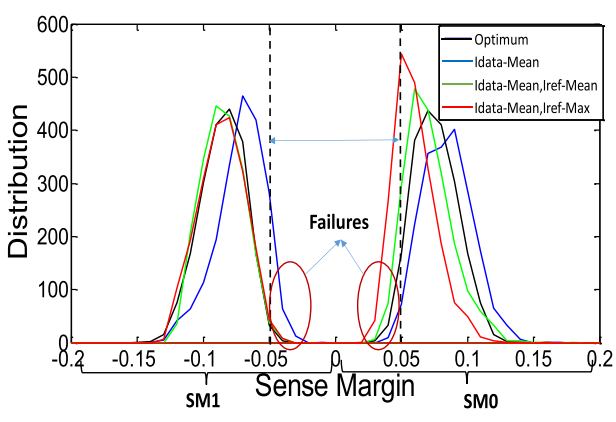

Fig. 10. Sense margin distribution for 2,000 Monte-Carlo points.

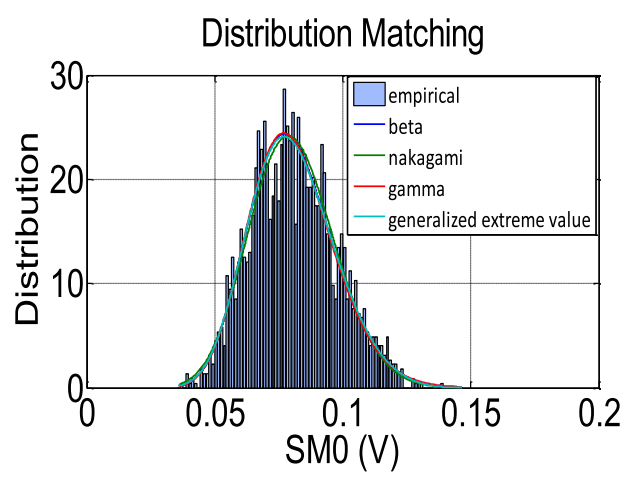

Fig. 11. SM0 distribution for 2,000 Monte-Carlo points. The curve fitting to model the tail is also shown.

Case (a) (Optimum): data and reference currents are bit-to-bit optimal to maximize the sense margins.

Case (b) ( $I_{\text {Data }}-$ Mean): the mean value of data current distribution is chosen as $\mathrm{I}_{\text {Data }}$ and reference current is the per-bit optimal current.

Case (c) $\left(I_{\text {Data }}-\right.$ Mean, $I_{\text {Ref }}-$ Max): the reference current is the worst case of the distribution and the data current is the mean value of distribution.

Case (d) (I $I_{\text {Data }}-$ Mean, $I_{R e f}-$ Mean): both data and reference current are the mean value of the current distribution.

Figure 10 shows the SM distribution for 2,000 Monte-Carlo points for cases (a) through (d). SM1 is negative, whereas SM0 is positive. An SA offset of $+/-50 \mathrm{mV}$ is assumed. As a result, SM1 greater than $-50 \mathrm{mV}$ and SM0 less than $50 \mathrm{mV}$ is considered as failure. Case (a) provides a distribution with the lowest standard deviation and higher mean value, which results in good sense margins for all bitcells and provides the minimum number of failures. We use case (a) to benchmark other cases. Case (d) is next best and is a good approximation of case (a).

In order to gain a detailed understanding, we use curve-fitting-based functions in Matlab to model the SM distribution (especially the tail). Figure 11 depicts different models (empirical, Extreme Value Theory, Beta, Nakagami, and Gamma) used to fit the distribution in Matlab. The empirical model indicated a better match for the tail. Therefore, we used this model for the cache-level analysis. Note that the number of bit cells for our study is $1 \mathrm{Mb}$. The curve-fitting model is used to extrapolate the distribution to $1 \mathrm{M}$ bits.

Figure 12(a) shows the number of SM0/SM1 failures after curve fitting for different cases assuming 50mV SA offset. Case (d) provides the lower number of failures compared to cases (b) and (c). Therefore, the mean value of reference and data current distribution is the best candidate for $I_{\text {Ref }}$ and $\mathrm{I}_{\text {Data. }}$. It can be noted that SM1 (SM0) failures are increased (decreased) in case (c) (case (d)). This is due to the fact that the maximum value of distribution is selected as the reference current, which increases the reference current (see Figure 7) and in turn decreases |SM1| (increases |SM0|).

Figure 12(b) shows total SM0 and SM1 failures with respect to SA offset voltage. Note that both case (a) and case (d) can sense all bitcells correctly below 25mV SA offset. High offset voltage of SA increases sense failures exponentially.

\section{READ CURRENT MODULATION}

In the previous section, we described the impact of variations and sense speed on sense failures. We concluded that the mean value of reference and data currents provides better sense margin. In 


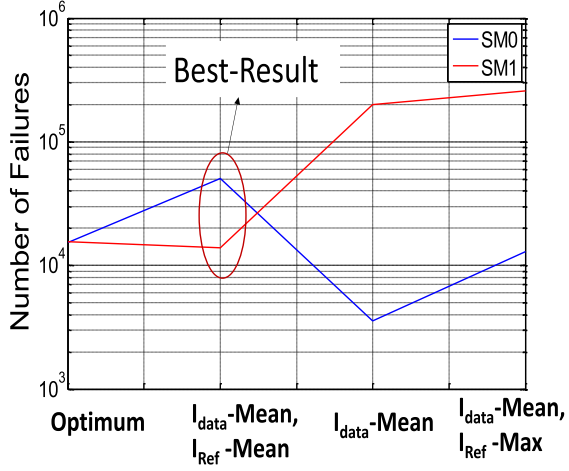

(a)

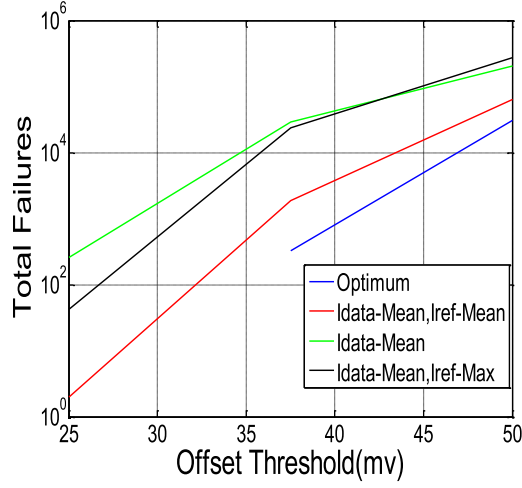

(b)

Fig. 12. (a) Number of failures for different cases of data and reference current selections for $1 \mathrm{M}$ bitcells where the SA offset voltage is $50 \mathrm{mV}$. (b) Total SM0 and SM1 failures with respect to SA offset voltage.

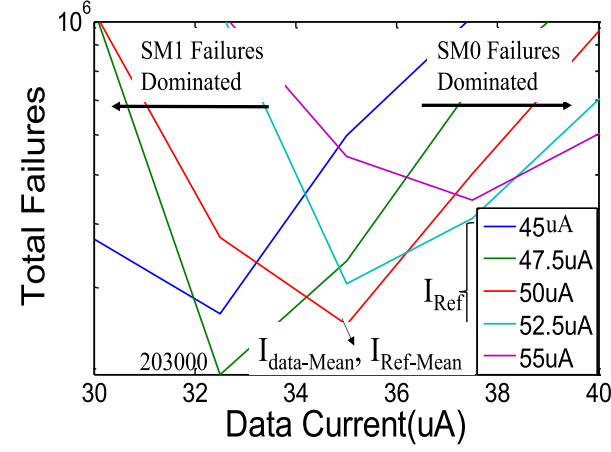

(a)

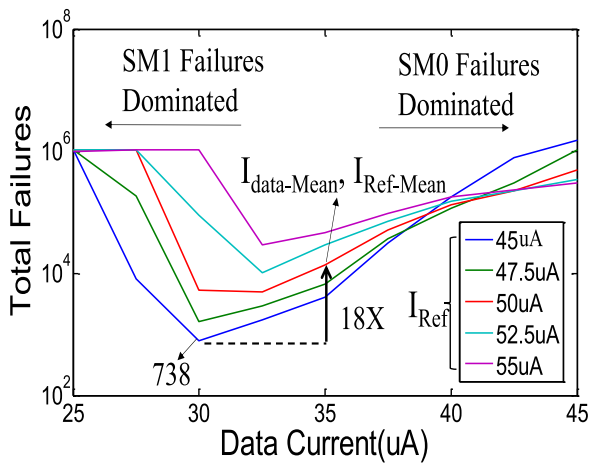

(b)

Fig. 13. Total SM0 and SM1 failures with respect to data and reference current variation for two SA offset voltages: (a) $50 \mathrm{mV}$ and (b) $25 \mathrm{mV}$.

this section, we show that small modulation of reference and data current around the mean value can optimize the failures further. This can be combined with sense time modulation as well.

\subsection{Reference and Data Current Modulation}

Figure 13(a) shows total SM0 and SM1 failures with respect to data and reference current variation for $50 \mathrm{mV}$ SA offset voltage. It can be noted that sense failure is sensitive to data and reference current selection. As shown in Figure 13, for each reference current there is a data current (nominal current) that provides minimum sensing failures. For data current lower than nominal current, total failures are dominated by SM1 failures, whereas for data current higher than nominal current, total failures are dominated by SM0 failures. In addition, case (d) does not always provide the minimum number of failures. Furthermore, optimized selection of data and reference current depends on SA offset voltage. For example, in Figure 13(b), choosing a data current of 30uA, instead of the mean value $(35 \mathrm{uA})$, causes an $18 \times$ reduction in sensing failures for $25 \mathrm{mV}$ offset voltage. Therefore, the reference and data current modulation capability for postsilicon adaptation can optimize the failures. 


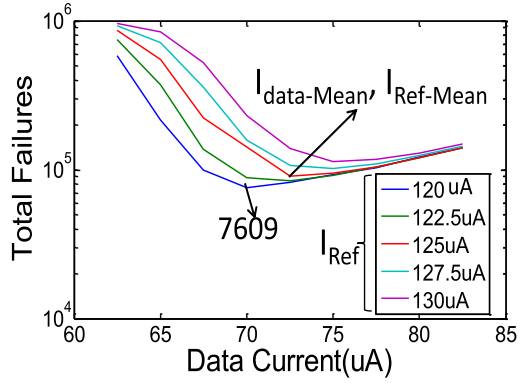

(a)

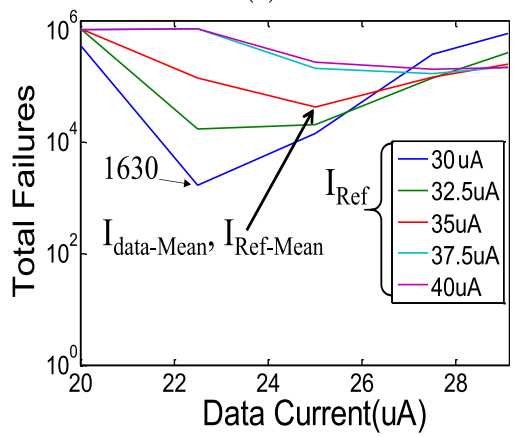

(c)

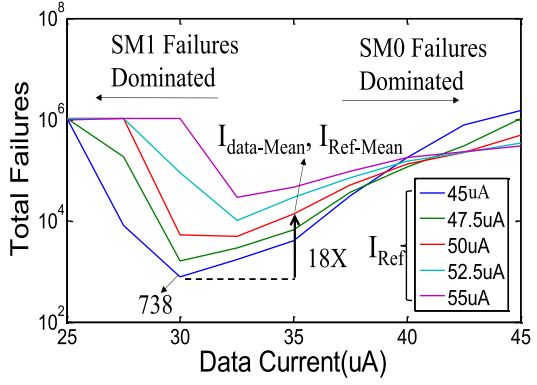

(b)

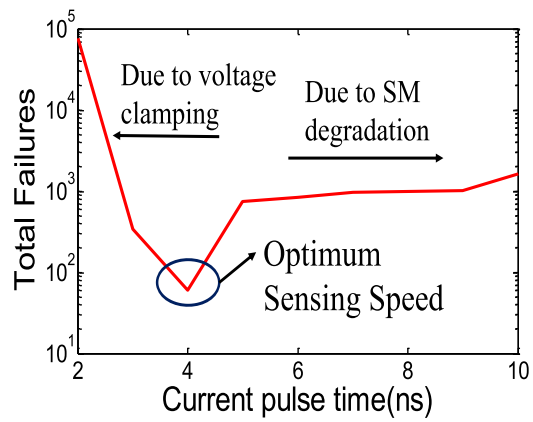

(d)

Fig. 14. Total SM0 and SM1 failures with respect to data and reference current variation for (a) $2 \mathrm{~ns}$, (b) $5 \mathrm{~ns}$, (c) $10 \mathrm{~ns}$ reference current pulse for $25 \mathrm{mV}$ offset voltage, and (d) total number of failures for different current pulse widths.

\subsection{Sense Time Modulation}

Figures 14(a) through 14(c) show total SM0 and SM1 failures with respect to data and reference current variation for $10 \mathrm{~ns}, 5 \mathrm{~ns}$, and $2 \mathrm{~ns}$ current pulses. Note that a $2 \mathrm{~ns}$ pulse results in an $\sim 100 \times$ increase in failures compared to the $4 \mathrm{~ns}$ pulse, due to more variation in data and reference current and clamping of sense voltage. A current pulse of $10 \mathrm{~ns}$ results in more failures compared to an $5 \mathrm{~ns}$ current pulse, due to lower sense current, which in turn results in poor sense margin. This is further illustrated in Figure 14(d), which plots the number of failures with respect to current pulse width. It is evident that pulse width modulation is necessary to optimize the sense failures. A 4ns current pulse width shows optimal failure in our simulation setup.

\subsection{Methodology}

The total number of failures depends on factors such as sense speed, SA offset, and data and reference current. We propose adaptive current magnitude and pulse width modulation to exploit these dependencies. The proposed current modulation is employed after a test routine that screens the SM1 and SM0 failures with mean reference and data current and pulse width. The test pattern can be a conventional March pattern (e.g., March C (Michael and Agrawal 2004)). From Figures 14(a) through (c), it is evident that lowering reference and data current simultaneously may result in lower sense failures. Furthermore, as depicted in Figure 14(d), we can modulate reference current pulse width to minimize sensing failures. We leverage these features to provide three configurations each for $\mathrm{I}_{\text {Data }}\left(\mathrm{I}_{\text {Data-Mean }}-\Delta_{\mathrm{D}}\right.$, $\mathrm{I}_{\text {Data-Mean }}$, $\left.\mathrm{I}_{\text {Data-Mean }}+\Delta_{\mathrm{D}}\right), \mathrm{I}_{\text {Ref }}\left(\mathrm{I}_{\text {Ref-Mean }}-\Delta_{\mathrm{R}}, \mathrm{I}_{\text {Data-Mean }}\right.$, $\left.\mathrm{I}_{\text {Ref-Mean }}+\Delta_{\mathrm{R}}\right)$, and reference current pulse $\left(\mathrm{t}_{\text {nom }}-\Delta_{\mathrm{T}}, \mathrm{t}_{\text {nom }}, \mathrm{t}_{\text {nom }}+\Delta_{\mathrm{T}}\right)$, which results in 27 total configurations. 


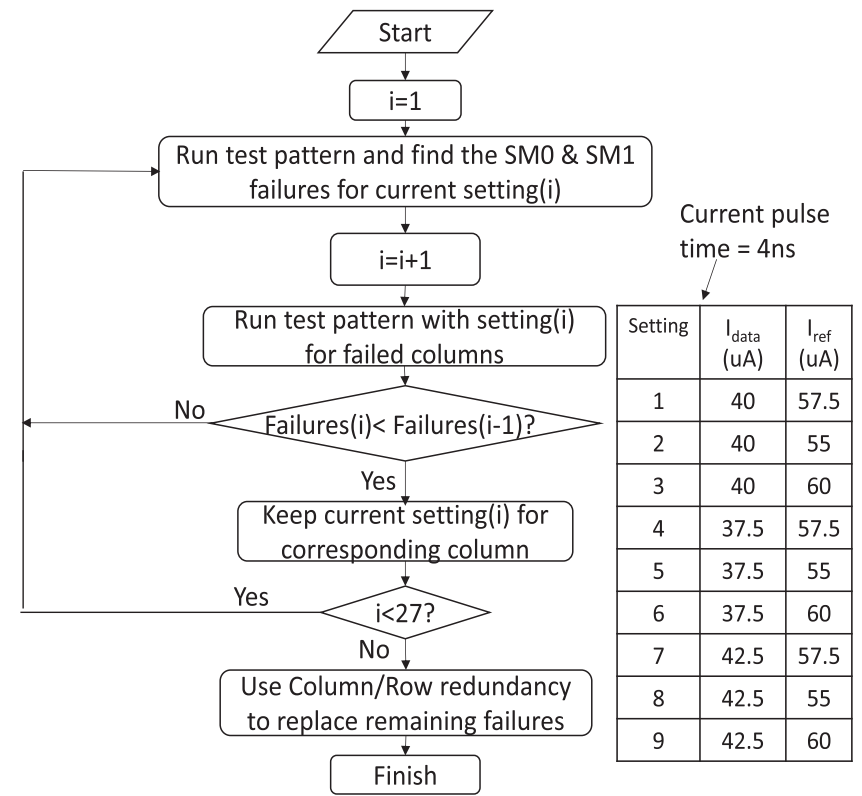

Fig. 15. Test pattern procedure to determine the best setting for data and reference currents.

Initially, we run a test pattern with $\mathrm{I}_{\text {Data-Mean }}, \mathrm{I}_{\text {Ref-Mean }}$, and $\mathrm{t}_{\text {mean }}$. The columns containing SM0/SM1 failures are marked individually. In this context, it is worth mentioning that the entire global column is marked SM0-related failure even if one of the local columns doesn't sense "0" correctly (we have eight local columns per global column). This is due to the fact that current sources are shared per global column basis. Next, the same patterns are repeated with another configuration to ensure that the selected column passes sensing correctly. We repeat this procedure till we find a setting with the least number of failures. The flowchart showing the proposed methodology is provided in Figure 15. For the failure columns, after applying the best setting, we suggest employing the existing column or row redundancies for replacement. The failure ratio can be reduced by using more settings for the read current modulation technique. In addition, error correction code (ECC) can be employed to further reduce the sensing failures. The test time is approximated as follows:

Test time $=\mathrm{N}_{\text {Rows }}{ }^{*}($ Write_latency + Read_latency $)+\mathrm{N}_{\text {Failing_columns }}{ }^{*}$ Nsetting ${ }^{*}\left(\right.$ read_latency $_{+}+$ mode_selection_latency),

where $\mathrm{N}_{\text {Row }}$ is the number of rows, and $\mathrm{N}_{\text {settings }}$ is the number of settings tried for each failing column in order to minimize the failures. We have considered both read and write latency for each row since each row is written and read subsequently during testing. From our estimates, the test time is in the order of milliseconds.

We performed 2,000 Monte-Carlo points in HSpice for different reference pulse widths and reference currents to obtain SM distribution for each configuration. The curve-fitting model in Matlab is used to extrapolate the distribution to $1 \mathrm{M}$ bits. Next, we modeled each global column to evaluate the read current modulation technique.

\subsection{Comparison with Conventional Sensing}

In order to compare proposed sensing with other sensing techniques, we tabulate the failure ratio $\left(100^{*}\right.$ failures/number of bitcells), power consumption, area overhead, and sense time. The 
Table 2. Comparison of Sensing Schemes

\begin{tabular}{|c|c|c|c|c|}
\hline & & & & $\begin{array}{c}\text { Failure } \\
\text { Ratio } \\
(25 \mathrm{mV} \\
\text { Sense Scheme }\end{array}$ \\
\hline $\begin{array}{c}\text { Nondestructive } \\
\text { voltage sensing }\end{array}$ & $1.03 \mathrm{um}^{2}$ & $80 \mathrm{uW}$ & $2.5 \mathrm{~ns}$ & $10.7 \%$ \\
\hline $\begin{array}{c}\text { Nondestructive } \\
\text { voltage sensing } \\
\text { (source-degen.) }\end{array}$ & $1.03 \mathrm{um}^{2}$ & $70 \mathrm{uW}$ & $2.5 \mathrm{~ns}$ & $6.9 \%$ \\
\hline $\begin{array}{c}\text { Destructive } \\
\text { self-ref. }\end{array}$ & $1.2 \mathrm{um}^{2}$ & $190 \mathrm{uW}$ & $9 \mathrm{~ns}$ & $1 \%$ \\
\hline $\begin{array}{c}\text { Destructive } \\
\text { self-ref. (adaptive) }\end{array}$ & $1.2 \mathrm{um}^{2}$ & $190 \mathrm{uW}$ & $9 \mathrm{~ns}$ & $0.01 \%$ \\
\hline
\end{tabular}

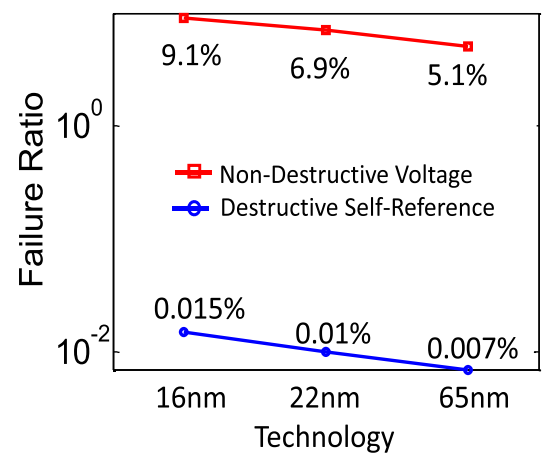

Fig. 16. Failure ratio trend for different technology processes.

comparison is depicted in Table 2. This result is obtained by redesigning and optimizing destructive self-reference and nondestructive voltage sensing schemes using $22 \mathrm{~nm}$ predictive technology.

The destructive self-reference sensing failure ratio (1\%) is significantly lower than nondestructive voltage sensing $(6.9 \%)$. Furthermore, adaptive current modulation reduces the failure ratio to $0.01 \%$. The sense speed and power consumption of the self-reference scheme is higher than conventional sensing due to two write steps. However, the self-reference sensing is acceptable in a large STTRAM array where robustness is more important than sense speed and power.

Note that the sense circuit areas reported in Table 2 are estimated for only one bitcell sensing. In order to estimate the sense circuit area, we sum all the transistors' areas and also considered the area required for local routing. Even though self-reference scheme area is more than nondestructive voltage sensing, it does not require any reference circuit, which incurs area overhead.

The effect of technology scaling on the failure ratio is shown in Figure 16. The failure ratio for nondestructive voltage sensing significantly increases as technology scales toward $16 \mathrm{~nm}$. However, technology scaling does not affect the failure ratio for the destructive self-reference scheme significantly. Therefore, the destructive self-reference scheme is promising for smaller technology processes.

We have performed the temperature and voltage variation analysis, and the result is shown in Figures 17(a) and 17(b). As shown in Figure 17(a), the proposed technique failure ratio reduces as the supply voltage increases. As supply voltage increases, the current injected into the bitcell during the read operation increases, which results in higher peak voltage (i.e., higher $|\mathrm{SM} 0|+$ $|S M 1|)$, as shown in Figure 9. Additionally, the voltage across MTJ is clamped at a higher voltage, which in turn improves the sense margin. In conventional sensing, all design parameters are optimized in such a way to provide the maximum SM at $1 \mathrm{~V}$ supply voltage. Therefore, an increase/decrease in supply voltage increases the sensing failure, as shown in Figure 17(a). Figure 17(b) depicts the failure ratio with respect to temperature variation. It can be noted that at higher and lower temperatures, the failure ratio increases. However, the proposed method shows significantly lower sensing failures under temperature variation. The proposed technique shows significantly lower sensing failures under supply voltage and temperature variation compared to conventional nondestructive sensing. 

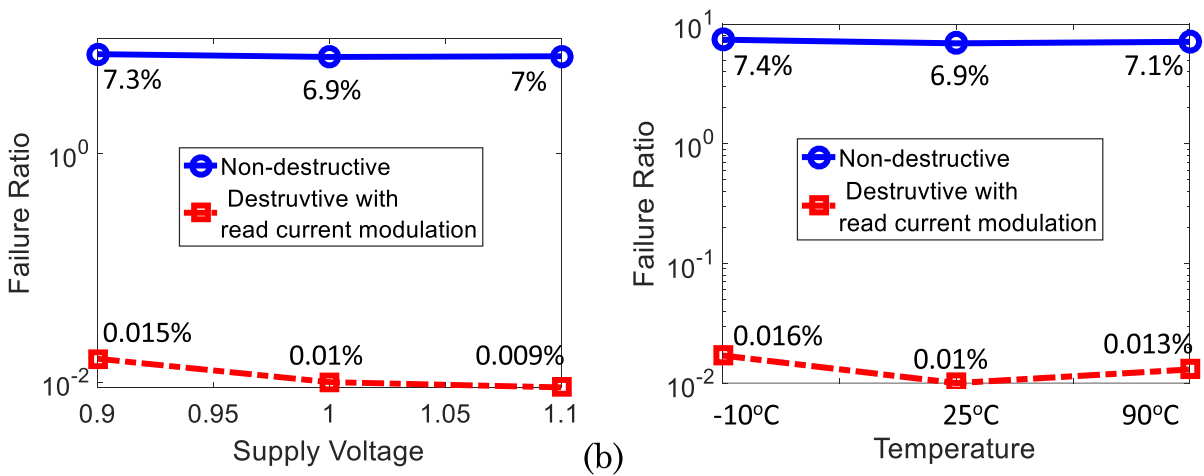

Fig. 17. Failure ratio trend for nondestructive voltage sensing with source degeneration and proposed destructive sensing with read current modulation under (a) supply voltage variation and (b) temperature variation.

\section{CONCLUSIONS}

In this work, we study the process variation effect on the self-reference sensing scheme to eliminate bit-to-bit process variation in MTJ resistance. Our investigation reveals that unoptimized selection of reference and data current can result in significant sensing failures. Furthermore, the sense speed is tightly coupled with the reference/data requirement. We propose read current modulation to overcome the failures due to process variation.

\section{REFERENCES}

ASU. Predictive Technology Model. 2008. Retrieved from http://www.asu.edu/ ptm.

K.S. Au Edward, Wing-Hung Ki, Wai Ho Mow, Silas T. Hung, and Catherine Y. Wong. 2004. A novel current-mode sensing scheme for magnetic tunnel junction MRAM. IEEE Transactions on Magnetics 40, 2 (2004), 483-488.

Lee Dongsoo and Kaushik Roy. 2014. Energy-delay optimization of the STT MRAM write operation under process variations. In IEEE Transactions on Nanotechnology 13, 4 (2014), 714-723.

Y. Emre et al. 2012. Enhancing the reliability of STT-RAM through circuit and system level techniques. In SiPS, 2012.

X. Fong, S. H. Choday, P. Georgios, C. Augustine, and K. Roy. 2013. SPICE Models for Magnetic Tunnel Junctions Based on Monodomain Approximation. Retrieved from https://nanohub.org/resources/19048.

D. Halupka et al. 2010. Negative-resistance read and write schemes for STT-MRAM in $0.13 \mu \mathrm{m}$ CMOS. In ISSCC, 2010.

M. Hosomi et al. 2005. A novel nonvolatile memory with spin torque transfer magnetization switching: Spin-RAM. In IEDM, 2005.

Gitae Jeong, Wooyoung Cho, Sujin Ahn, Hongsik Jeong, Gwanhyeob Koh, Youngnam Hwang, and Kinam Kim. 2003. A 0.24-/spl mu/m 2.0-V 1T1MTJ 16-kb nonvolatile magnetoresistance RAM with self-reference sensing scheme. In IEEE Journal of Solid-State Circuits 38, 11 (2003), 1906-1910.

L. Jing et al. 2008. Variation-tolerant spin-torque transfer (STT) MRAM array for yield enhancement. In IEEE Custom Integrated Circuits Conference, 2008 (CICC'08). IEEE.

K. Jisu et al. 2012. A novel sensing circuit for deep submicron spin transfer torque MRAM (STT-MRAM). IEEE Transactions on Very Large Scale Integration (VLSI) Systems 20, 1 (2012), 181-186.

K. Jisu et al. 2014. STT-MRAM sensing circuit with self-body biasing in deep submicron technologies. IEEE Transactions on Very Large Scale Integration (VLSI) Systems 22, 7 (2014), 1630-1634.

A. Junwhan, S. Yoo, and K. Choi. 2014. DASCA: Dead write prediction assisted STT-RAM cache architecture. In 2014 IEEE 20th International Symposium on High Performance Computer Architecture (HPCA'14). IEEE.

A. Khouzani, H. Y. Xue, C. Yang, and A. Pandurangi. 2014. Prolonging PCM lifetime through energy-efficient, segmentaware, and wear-resistant page allocation. In Proceedings of the 2014 International Symposium on Low Power Electronics and Design. ACM, 327-330.

Y. Kim et al. 2012. Write-optimized reliable design of STT MRAM. In ISLPED, 2012.

D. Lee et al. 2012. High-performance low-energy STT MRAM based on balanced write scheme. In ISLPED, 2012.

J. Li et al. 2010. Design paradigm for robust spin-torque transfer magnetic RAM (STT MRAM) from circuit/architecture perspective. In TVLSI, 2010 
Marcel J. M. Pelgrom, Aad C. J. Duinmaijer, and Anton P. G. Welbers. 1989. Matching properties of MOS transistors. IEEE fournal of Solid-State Circuits 24, 5 (1989): 1433-1439.

Bushnell Michael and Vishwani Agrawal. 2004. Essentials of electronic testing for digital, memory and mixed-signal VLSI circuits. 17. Springer Science \& Business Media.

I. Mohsen, S. Patil, and T. Rosing. 2016. Low power data-aware STT-RAM based hybrid ache architecture. In 2016 17th International Symposium on Quality Electronic Design (ISQED'16). IEEE.

I. Mohsen et al. 2016. A low-power hybrid magnetic cache architecture exploiting narrow-width values. In 2006 5th NonVolatile Memory Systems and Applications Symposium (NVMSA'16). IEEE.

Zand Ramtin, Arman Roohi, and Ronald F. DeMara. 2017. Energy-efficient and process-variation-resilient write circuit schemes for spin hall effect MRAM Device. In IEEE Transactions on Very Large Scale Integration (VLSI) Systems.

M. Rasquinha et al. 2010. An energy efficient cache design using spin torque transfer (stt) ram. In ISLPED, 2010.

F. Ren et al. 2012. A body-voltage-sensing-based short pulse reading circuit for spin-torque transfer RAMs (STT-RAMs). In ISQED, 2012

J. Seong-Ook et al. 2011. Balancing a signal margin of a resistance based memory circuit. U.S. Patent 7, 889, 585. Feb. 15, 2011.

M. Seyedhamidreza and S. Ghosh. 2014. Simultaneous sizing, reference voltage and clamp voltage biasing for robustness, self-calibration and testability of STTRAM arrays. In Proceedings of the 51st Annual Design Automation Conference. ACM, $1-2$.

M. Seyedhamidreza et al. 2015. A novel slope detection technique for robust STTRAM sensing. In 2015 IEEE/ACM International Symposium on Low Power Electronics and Design (ISLPED'15). IEEE, 7-12.

C. W. Smullen et al. 2011. Relaxing non-volatility for fast and energy-efficient STT-RAM caches. In HPCA, 2011.

Jee-Hwan Song, Jisu Kim, Seung H. Kang, Sei-Seung Yoon, and Seong-Ook Jung. 2011. Sensing margin trend with technology scaling in MRAM. In International fournal of Circuit Theory and Applications 39, 3 (2011), 313-325.

C. Xu et al. 2010. Device-architecture co-optimization of STT-RAM based memory for low power embedded systems. In ICCAD, 2010.

Z. Yaojun et al. 2011. STT-RAM cell optimization considering MTJ and CMOS variations. IEEE Transactions on Magnetics 47, 10 (2011), 2962-2965.

C. Yiran et al. 2012. A $130 \mathrm{~nm} 1.2 \mathrm{~V} / 3.3 \mathrm{~V} 16 \mathrm{~Kb}$ spin-transfer torque random access memory with nondestructive selfreference sensing scheme. IEEE fournal of Solid-State Circuits 47, 2 (2012), 560-573.

P. Zhou et al. 2009. Energy reduction for STT-RAM using early write termination. In ICCAD, 2009.

Received January 2016; revised May 2017; accepted August 2017 